\title{
Quantum Metamaterials with Magnetic Response at Optical Frequencies
}

\author{
Rasoul Alaee $\odot,{ }^{1,2, *}$ Burak Gurlek®, ${ }^{1,3}$ Mohammad Albooyeh $\odot,{ }^{4}$ Diego Martín-Cano $\odot,{ }^{1}$ and Vahid Sandoghdar $\odot^{1,3, \dagger}$ \\ ${ }^{1}$ Max Planck Institute for the Science of Light, Erlangen 91058, Germany \\ ${ }^{2}$ Department of Physics, University of Ottawa, Ottawa Q1N 6N5, Canada \\ ${ }^{3}$ Department of Physics, Friedrich Alexander University Erlangen-Nuremberg, D-91052 Erlangen, Germany \\ ${ }^{4}$ Department of Electrical Engineering and Computer Science, University of California, Irvine, California 92617, USA
}

(Received 10 February 2020; accepted 10 July 2020; published 7 August 2020)

\begin{abstract}
We propose novel quantum antennas and metamaterials with a strong magnetic response at optical frequencies. Our design is based on the arrangement of natural quantum emitters with only electric dipole transition moments at distances smaller than a wavelength of light but much larger than their physical size. In particular, we show that an atomic dimer can serve as a magnetic antenna at its antisymmetric mode to enhance the decay rate of a magnetic transition in its vicinity by several orders of magnitude. Furthermore, we study metasurfaces composed of atomic bilayers with and without cavities and show that they can fully reflect the electric and magnetic fields of light, thus, forming nearly perfect electric or magnetic mirrors. The proposed metamaterials will embody the intrinsic quantum functionalities of natural emitters such as atoms, ions, color center, or molecules and can be fabricated with available state-of-the-art technologies, promising several applications both in classical optics and quantum engineering.
\end{abstract}

DOI: 10.1103/PhysRevLett.125.063601

Most natural materials interact weakly with the magnetic field of light at optical frequencies [1]. In fact, the magnetic interaction energy $\boldsymbol{- \mu} \cdot \mathbf{B}$ is typically about 2 orders of magnitude (i.e., order of fine-structure constant) smaller than its electric counterpart $-\mathbf{p} \cdot \mathbf{E}$, whereby $\mu \approx \mu_{B}$ and $p \approx$ $e a_{0}$ represent the magnitude of the magnetic and electric dipole moments, and $e, a_{0}, \mu_{B}$ denote the elementary charge, Bohr radius, and Bohr magneton, respectively [2]. However, two decades of progress in nano-optics [3] has brought about novel electromagnetic properties that are not available in natural materials. In particular, "metamaterials" created through synthetic arrangement of subwavelength antennas [4-7] can now generate magnetic functionalities at high frequencies [8]. Unfortunately, material absorption and limits in nanofabrication have hampered reaching a high performance in the optical regime.

Considering that natural atoms act as the smallest and most fundamental optical antennas $[9,10]$, one can also envision the construction of novel metamaterials by synthetically arranging atoms, ions, color centers, or molecules with intrinsic quantum properties. In this approach, one assembles individual quantum emitters at distances smaller than an optical wavelength but much larger than the characteristic length of electronic orbitals. Indeed, a number of proposals have emerged over the past few years for synthetic arrangement of natural atoms [11-26], but these have only considered metamaterials with electric response. In this Letter, we show that a strong magnetic functionality can be obtained from conventional quantum emitters at optical frequencies. In particular, we propose novel quantum antennas that can enhance the decay rate of a magnetic emitter (i.e., an emitter with magnetic dipole transitions) in their vicinity by several orders of magnitude. We demonstrate that a metasurface composed of the proposed antennas can act as nearly perfect electric and magnetic mirrors and can, moreover, strongly couple to a cavity mode independent of its position. In what follows, we will use the terms "atoms" and "quantum emitters" interchangeably.

Atomic dimer antenna.-First, let us consider an atomic dimer consisting of two identical atoms with electric dipole transition moments placed at $\mathbf{r}_{u / d}=(0,0, \pm l / 2)$ [see the inset in Figs. 1(a) and 1(b) and Fig. 2(b); $u, d$ stand for up and down]. We assume that the atomic response is isotropic and linear, i.e., we consider the weak-excitation limit. The electric polarizability of each atom amounts to $\alpha(\omega)=\left[-\left(\Gamma_{0} / 2\right) \alpha_{0}\right] /\left[\delta+i\left(\Gamma_{0} / 2\right)\right]$, where $\Gamma_{0}$ is the radiative linewidth of the atomic transition at frequency $\omega_{a}$ while $\delta=\omega-\omega_{a} \ll \omega_{a}$ represents the frequency detuning between the illumination and the atom, $\alpha_{0}=6 \pi / k^{3}$ and $k$ is the wave number $[27,28]$.

The response of the atomic dimer can be computed by considering their dipole-dipole interaction. For convenience, in analytical calculations, we consider illumination by an $x$-polarized plane wave $\mathbf{E}_{\text {inc }}=E_{0} e^{i k z} \mathbf{e}_{x}$ propagating in the $z$ direction, where $\mathbf{e}_{x}$ is the unit vector in the $x$ direction, $E_{0}$ is the electric field amplitude, and $k$ is the wave number in free space. Using coupled dipole theory [29,30], we obtain the effective induced electric dipole moments $\mathbf{p}_{u / d}=\epsilon_{0} \alpha_{u / d} \mathbf{E}_{\text {inc }}$ in the upper and lower atoms of the dimer antenna where $\alpha_{u / d}=\alpha\left\{\left[\cos (k l / 2) / D_{-}\right] \pm i\left[\sin (k l / 2) / D_{+}\right]\right\} e^{\mp i k l / 2}$ are the effective electric polarizabilities of the upper and lower 

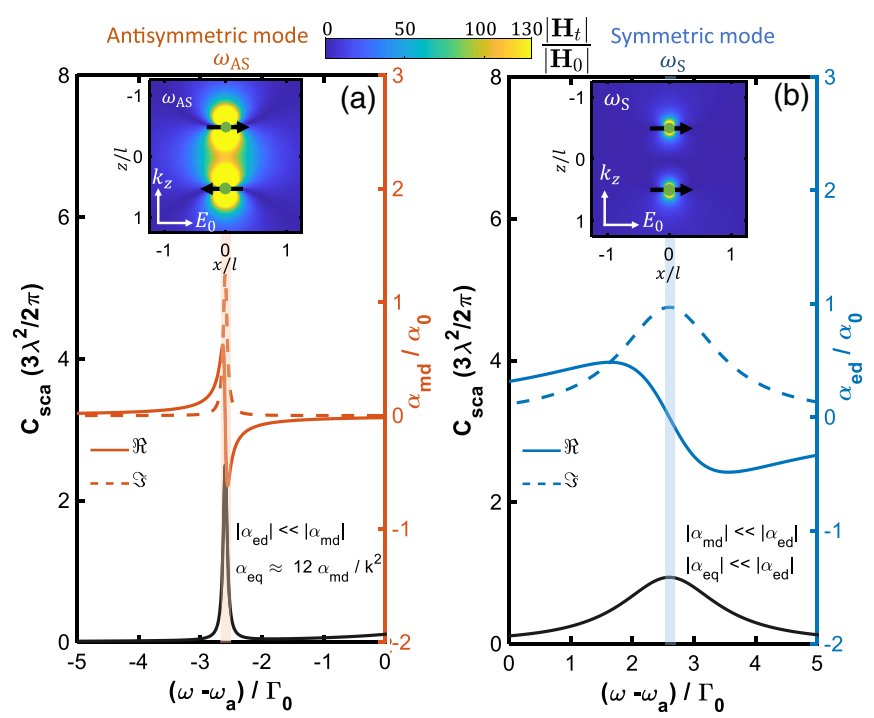

FIG. 1. Atomic dimer antenna: Optical response of two atoms placed at $z= \pm l / 2$ (see insets) as a function of detuning at the antisymmetric (AS) (a) and symmetric (b) modes of the composite system, respectively. Left vertical axes (black): total scattering cross sections normalized to the free-space value $3 \lambda^{2} / 2 \pi$ for a two-level atom on resonance. Right vertical axes (orange and blue): The real (solid curves) and imaginary (dashed curves) parts of the induced effective polarizabilities calculated using Eq. (1) and for $l=0.1 \lambda_{a}$, where $\lambda_{a}$ is the wavelength detuning between the illumination and the atom. Insets display normalized total magnetic field distribution for each case, where $\mathbf{H}_{t}, \mathbf{H}_{0}$ are the total and incident magnetic fields, respectively.

atoms [31]. Here, $D_{ \pm} \equiv 1 \pm \epsilon_{0} \alpha G_{\mathrm{EE}}^{x x}\left(\mathbf{r}_{u}, \mathbf{r}_{d}\right)$ signify the interdependent effect of the atoms via a scalar Green's function $G_{E E}^{x x}\left(\mathbf{r}_{u}, \mathbf{r}_{d}\right)$ of the Helmholtz equation in free space [2]. The induced dipole moments can be used to express an induced current density, which can be expanded in multipoles [37,38], leading to the induced electric and magnetic dipole and quadrupole polarizabilities of the dimer antenna at $\mathbf{r}=0[31]$
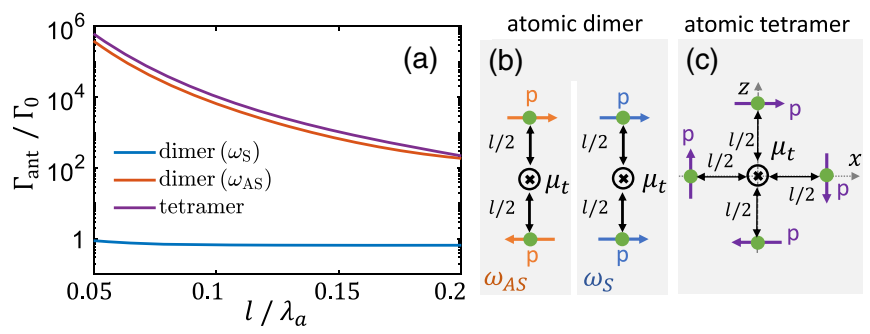

FIG. 2. Enhancing the decay rate of a magnetic transition: (a) Enhanced decay rate of a magnetic dipole emitter placed in the middle of an atomic dimer antenna for the symmetric (blue), antisymmetric (red) modes and an atomic tetramer (purple) as a function of the antenna length $l$. The tetramer antenna is composed of four identical atoms placed at $\mathbf{r}_{1,2}=\mp l / 2 \mathbf{e}_{z}, \mathbf{r}_{3,4}= \pm l / 2 \mathbf{e}_{x}$. (b),(c) Schematics of an emitter with magnetic dipole moment $\mu_{t}$ placed in the middle of an atomic dimer (b) and tetramer (c).

$$
\begin{aligned}
\alpha_{\mathrm{ed}} & =\frac{2 \alpha}{D_{-}}\left[j_{0}(k l / 2)-\frac{j_{2}(k l / 2)}{2}\right] \cos (k l / 2), \\
\alpha_{\mathrm{md}} & =\frac{3 \alpha}{D_{+}} j_{1}(k l / 2) \sin (k l / 2), \\
\alpha_{\mathrm{eq}} & =\frac{12}{k^{2}} \frac{\alpha}{D_{+}}\left[3 j_{1}(k l / 2)-2 j_{3}(k l / 2)\right] \sin (k l / 2), \\
\alpha_{\mathrm{mq}} & =-\frac{60}{k^{2}} \frac{\alpha}{D_{-}} j_{2}(k l / 2) \cos (k l / 2) .
\end{aligned}
$$

Here, $j_{n}(r)$ is the spherical Bessel function and $\alpha_{\mathrm{ed}}, \alpha_{\mathrm{md}}, \alpha_{\mathrm{eq}}$, and $\alpha_{\mathrm{mq}}$ represent the electric dipole, magnetic dipole, electric quadrupole, and magnetic quadrupole polarizabilities, respectively. For small separations $(l \ll \lambda)$, the higher order spherical Bessel functions are negligible, i.e., $j_{1}(k l / 2) \approx 1, j_{2}(k l / 2) \approx 0$, and $j_{3}(k l / 2) \approx 0$. Thus, $\alpha_{\text {md }} \approx$ $k^{2} / 12 \alpha_{\text {eq }}$ and the magnetic quadrupole polarizability can be neglected, i.e., $\alpha_{\mathrm{mq}} \approx 0$. Once the induced dipole and quadrupole polarizabilities are obtained, the total scattering cross section $\left(C_{\text {sca }}\right)$ of the atomic dimer can also be calculated [37]

$$
C_{\text {sca }}=\frac{k^{4}}{6 \pi}\left(\left|\alpha_{\mathrm{ed}}\right|^{2}+\left|\alpha_{\mathrm{md}}\right|^{2}+\frac{3}{5}\left|\frac{k^{2}}{12} \alpha_{\mathrm{eq}}\right|^{2}\right) .
$$

Near-field coupling of the electric dipole transitions of two individual emitters has been explored in various systems [39,40] and is known to lead to symmetric (superradiant) and antisymmetric (subradiant) states. The black curve in Fig. 1(a) shows $C_{\text {sca }}$ for the subradiant state as a function of the frequency detuning for two atoms separated by $l=0.1 \lambda_{a}$. In this case, the electric response of the dimer antenna becomes negligible, but it exhibits both magnetic dipolar (see right vertical axis) and electric quadrupolar responses with $\alpha_{\mathrm{eq}} \approx\left(12 / k^{2}\right) \alpha_{\mathrm{md}}$ [31]. The inset in Fig. 1(a) illustrates the magnetic field distribution for this antisymmetric mode under plane wave illumination, where a strong magnetic field testifies to an optically induced magnetic response. The left vertical axis in Fig. 1(b) shows $C_{\text {sca }}$ as a function of the frequency detuning for the symmetric mode, where the two atoms oscillate in phase. The right vertical axis plots the electric dipolar response of the antenna structure, while the inset shows that the magnetic response is negligible in this scenario. We also investigated the effect of polarization and angle of incidence. The results can be found in the Supplemental Material (SM) [31].

Enhancing magnetic transitions.-The strong magnetic field generated in the atomic dimer [see Fig. 1(a)] prompts us to inquire whether it can act as a magnetic antenna to enhance the decay of a test magnetic dipole moment $\mu_{t}$ placed at the origin. Using the normalized local density of states of the system, one can arrive at the antenna-modified decay rate $\Gamma_{\text {ant }}$ given by [31] 


$$
\frac{\Gamma_{\mathrm{ant}}}{\Gamma_{0}}=1-\epsilon_{0}^{2} \alpha_{0} \alpha \operatorname{Im}\left[\frac{g_{\mathrm{EM}}^{2}\left(\mathbf{r}_{0}, \mathbf{r}_{u}\right)+g_{\mathrm{EM}}^{2}\left(\mathbf{r}_{0}, \mathbf{r}_{d}\right)}{1+\epsilon_{0} \alpha G_{\mathrm{EE}}^{x}\left(\mathbf{r}_{u}, \mathbf{r}_{d}\right)}\right],
$$

where $g_{\mathrm{EM}}\left(\mathbf{r}, \mathbf{r}^{\prime}\right)=-\left(3 / 2 \epsilon_{0} \alpha_{0}\right) e^{i \zeta}\left(1 / \zeta+i / \zeta^{2}\right)$ is the scalar electro-magnetic Green's function in free space and $\zeta=$ $k\left|\mathbf{r}-\mathbf{r}^{\prime}\right|[2,41]$. Figure 2(a) plots the calculated magnetic decay rate enhancement for both symmetric and antisymmetric modes. We find that the decay rate can be enhanced by 5 orders of magnitude at $l \approx 0.05 \lambda_{a}$ for the antisymmetric mode. We note that, as indicated by the distribution of the local magnetic field shown in the inset of Fig. 1(a), the observed enhancement is not restricted to the antenna center (see SM [31]). For the symmetric mode, the decay rate is even slightly decreased below its unperturbed value (i.e., $\Gamma_{\text {ant }}<\Gamma_{0}$ ) because of the weak magnetic response of this mode.

To achieve even larger enhancements, one can devise an atomic tetramer antenna, consisting of four identical atoms with electric polarizability $\alpha$ [see Fig. 2(c)]. Figure 2(a) shows the enhancement of the magnetic transition rate for this case [31]. We note that fabrication of quantum metamaterials in these configurations is readily within reach since the distances involved are well beyond atomic and molecular spacings in natural substances (e.g., $0.05 \lambda_{a}$ corresponds to several tens of nanometers). A particularly interesting class of materials for these applications is rare earth ions with weak magnetic dipole transition $[42,43]$. New efforts on the implantation of ions using ion traps or other bombardment strategies $[44,45]$ allow precise doping of various host materials. Furthermore, emerging techniques for the manipulation of neutral atoms might also be promising to explore [46-48].

Electric and magnetic mirrors based on atomic bilayer metasurfaces.-It has been shown that optimal optical coupling to a two-level atom requires mode matching between the incident light and that of the field radiated by the atom [9]. Thus, it is found that a dipolar wave can be perfectly reflected by a single two-level atom with a dipolar transition. Similarly, it has been shown, both theoretically and experimentally, that a planar two-dimensional array of atoms acts as a nearly perfect electric mirror for a planewave illumination $[12,13,24-26]$. Now, we show that a periodic planar arrangement of our dimer antennas, which we call atomic bilayer metasurfaces (ABM), can act as both electric and magnetic mirrors [see Fig. 3(a)].

To arrive at the reflected and transmitted fields by an $\mathrm{ABM}$ under illumination by a plane wave $\mathbf{E}_{\mathrm{inc}}=E_{0} e^{i k z} \mathbf{e}_{x}$, we first calculated the effective electric dipole moments of the upper and lower layers, $p_{u}^{\text {eff }}$ and $p_{d}^{\text {eff }}[29,30]$

$$
\left[\begin{array}{c}
p_{d}^{\text {eff }} \\
p_{u}^{\text {eff }}
\end{array}\right]=\left[\begin{array}{cc}
\frac{1}{\epsilon_{0} \alpha}-C_{d d} & -C_{d u} \\
-C_{u d} & \frac{1}{\epsilon_{0} \alpha}-C_{u u}
\end{array}\right]^{-1}\left[\begin{array}{c}
E_{\mathrm{inc}}\left(\mathbf{r}_{d}\right) \\
E_{\mathrm{inc}}\left(\mathbf{r}_{u}\right)
\end{array}\right]
$$

with

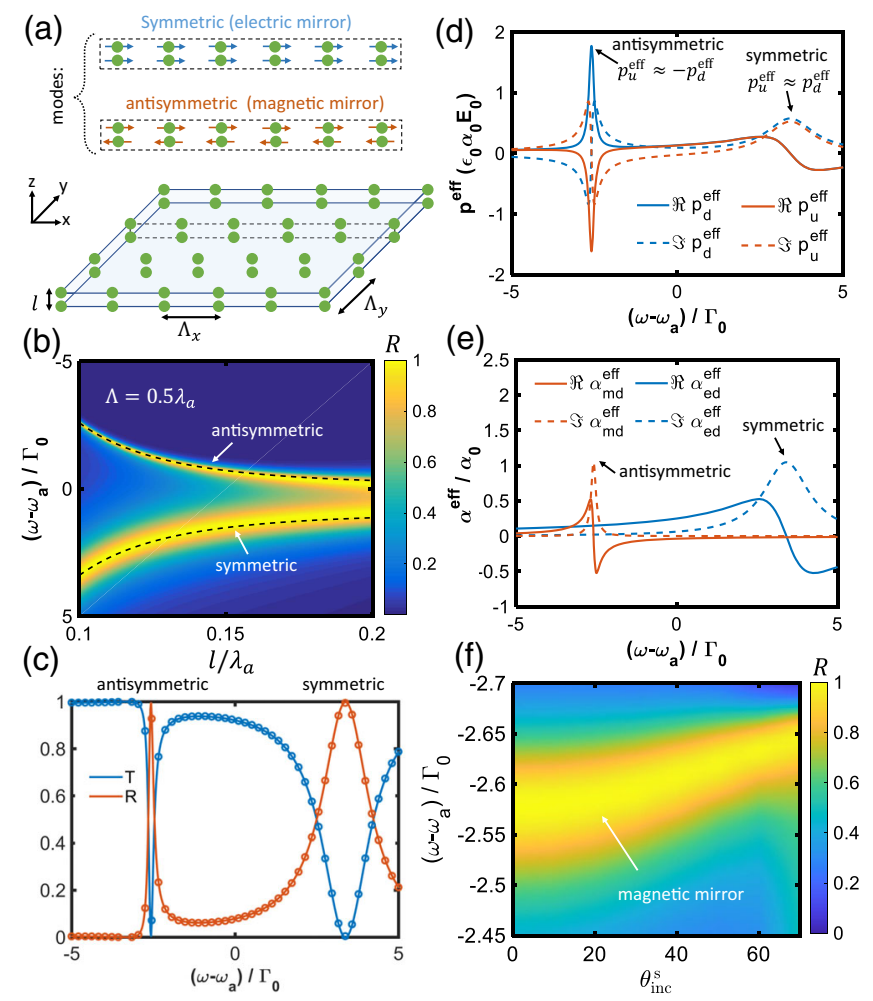

FIG. 3. Atomic bilayer metasurface (ABM): (a) Schematics of an ABM. The symmetric and antisymmetric modes corresponding to the electric and magnetic mirrors, respectively. (b) Intensity reflection coefficient $R$ as a function of layer separation $l$ and frequency detuning $\omega-\omega_{a}$ for an ABM with period $\Lambda_{x}=\Lambda_{y}=\Lambda=0.5 \lambda_{a}$. (c) Intensity transmission and reflection coefficients corresponding to a cut through (b) at $l=0.1 \lambda_{a}$. Solid curves: analytical results for an infinite array with plane wave illumination. Symbols: Numerical calculations for a finite array with $15 \times 15 \times 2$ atoms illuminated by a Gaussian beam. (d) Real and imaginary parts of the effective induced dipole moments of different layers. (e) Real and imaginary parts of the effective electric and magnetic polarizabilities. (f) Intensity reflection coefficient as a function of angle of incidence $\theta_{\text {inc }}^{s}$ (for s-polarized light) and frequency detuning for the antisymmetric mode.

$$
\begin{aligned}
C_{d d} & =\sum_{n, n \neq 0} G_{\mathrm{EE}}^{x x}\left(\mathbf{r}_{d, 0}, \mathbf{r}_{d, n}\right), \\
C_{d u} & =\sum_{n} G_{\mathrm{EE}}^{x x}\left(\mathbf{r}_{d, 0}, \mathbf{r}_{u, n}\right) .
\end{aligned}
$$

For identical atoms, the interaction constants are symmetric, i.e., $C_{u d}=C_{d u}$ and $C_{u u}=C_{d d}$. For atoms with unity quantum efficiency, the imaginary part of the interaction constants can be calculated exactly by using the conservation of energy principle $[49,50]$,

$$
\begin{aligned}
& \operatorname{Im}\left[C_{d d}\right]=\frac{k}{2 \Lambda^{2} \epsilon_{0}}-\frac{1}{\epsilon_{0} \alpha_{0}}, \\
& \operatorname{Im}\left[C_{d u}\right]=\frac{k}{2 \Lambda^{2} \epsilon_{0}} \cos (k l),
\end{aligned}
$$


while their real parts are calculated numerically. Having formulated the response of the ABM, the reflected and transmitted electric fields can now be obtained [31]

$$
\begin{aligned}
& r=\frac{i k}{2 \Lambda^{2} \epsilon_{0} E_{0}}\left(p_{d}^{\mathrm{eff}} e^{-i k l / 2}+p_{u}^{\mathrm{eff}} e^{i k l / 2}\right), \\
& t=1+\frac{i k}{2 \Lambda^{2} \epsilon_{0} E_{0}}\left(p_{d}^{\mathrm{eff}} e^{i k l / 2}+p_{u}^{\mathrm{eff}} e^{-i k l / 2}\right) .
\end{aligned}
$$

Figure 3(b) shows the reflectivity of an $\mathrm{ABM}$ as a function of the frequency detuning and the distance between the two layers. It can be seen that the array fully reflects the impinging light at both symmetric and antisymmetric resonance frequencies. In Fig. 3(c), we plot the reflection and transmission of a plane wave incident on an infinite array for $l=0.1 \lambda_{a}$ calculated using Eq. (9) (solid lines). The symbols in Fig. 3(c) present the results obtained for a finite array of $15 \times 15 \times 2$ atoms and a Gaussian beam illumination (a possible experimental situation) by integrating the Poynting vector for the scattered and incident beams [31]. We find that the results for the Gaussian and plane wave illuminations agree very well. We remark that, as in the case of atomic monolayer metasurfaces (AMMs) $[13,24]$, our findings for ABMs hold for all lattice periods smaller than $\lambda$ and are quite robust against disorder (see SM [31]).

Now, we provide more insight into the working of the ABM in Figs. 3(d) and 3(e). As displayed in Fig. 3(d), the effective induced dipole moments of the upper and lower layers are out of phase at the antisymmetric mode, i.e., $p_{u}^{\text {eff }} \approx-p_{d}^{\text {eff }} \approx 1.7 \epsilon_{0} \alpha_{0} E_{0}$. Figure 3(e) displays the effective electric and magnetic polarizabilities of the atomic metasurface, which can be calculated by using

$$
\begin{aligned}
& \alpha_{\mathrm{ed}}^{\mathrm{eff}}=\frac{p_{d}^{\mathrm{eff}}+p_{u}^{\mathrm{eff}}}{\epsilon_{0} E_{0}} \cos (k l / 2), \\
& \alpha_{\mathrm{md}}^{\mathrm{eff}}=i \frac{p_{d}^{\mathrm{eff}}-p_{u}^{\mathrm{eff}}}{\epsilon_{0} E_{0}} \sin (k l / 2) .
\end{aligned}
$$

It follows that the antisymmetric resonance of the ABM supports an effective magnetic response $\alpha_{\mathrm{md}}^{\text {eff }} \approx i \alpha_{0}$, while $\alpha_{\mathrm{ed}}^{\mathrm{eff}} \approx 0$. Together with a reflectivity $r \approx 1$, this implies that the bilayer metasurface acts as a nearly perfect atomic magnetic mirror at its antisymmetric resonance. At the symmetric mode, however, the atoms in the upper and lower layers are in phase such that $p_{u}^{\text {eff }} \approx p_{d}^{\text {eff }} \approx 0.5 i \epsilon_{0} \alpha_{0} E_{0}$, leading to an effective electric response, i.e., $\alpha_{\mathrm{ed}}^{\mathrm{eff}} \approx i \alpha_{0}$, but $\alpha_{\mathrm{md}}^{\text {eff }} \approx 0$ [see Fig. 3(e)]. Therefore, the array acts as a nearly perfect atomic electric mirror with $r \approx-1$. Our results are quite robust against changes in the angle of incidence, polarization, and the mode of the illuminating beam [see Fig. 3(f) and SM [31]].

Metasurfaces in a cavity.-Optical cavities are commonly used to enhance the interaction of light with matter.
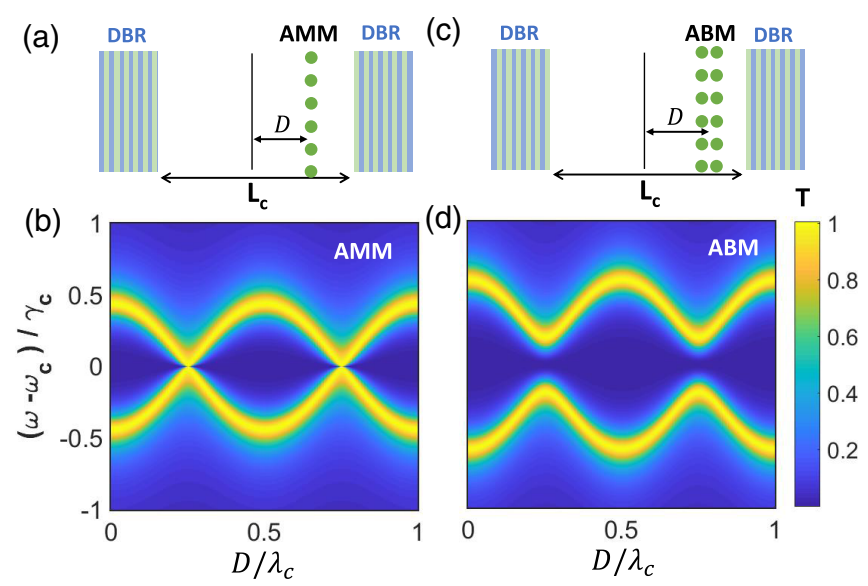

FIG. 4. Atomic metasurface inside a planar cavity composed of distributed Bragg reflectors (DBRs): (a) Schematics of a cavity and an atomic monolayer metasurface (AMM). (b) Transmission of the cavity loaded with the AMM shows resonance splitting as a function of its position inside the cavity $(D)$. (c),(d) Same as (a), (b) but for an atomic bilayer metasurface (ABM). The quality factor of the planar cavity is taken to be $Q \approx 8.7 \times 10^{3}, \gamma_{c} \approx$ $10^{3} \Gamma_{0}$ and $L_{c}=3 \lambda_{a}$.

The maximum interaction occurs when an atom is placed at the maximum of the cavity field, i.e., the interaction strongly depends on the position of the atom. The combination of the strong electric and magnetic response of the proposed atomic bilayer metasurface can relax this restriction.

Figure 4 depicts a planar cavity consisting of two mirrors separated by $L_{c}$, whereby the transmission of the bare cavity is assumed to reach unity at $\omega=\omega_{c}$. Now, we place an atomic metasurface at a distance $D$ from the cavity center [see Figs. 4(a) and 4(c)]. As in the case of a single atom, we find that the transmission of the cavity containing an AMM depends sensitively on its position $D$ [see Fig. 4(b)]. In particular, strong coupling can be reached at $D=0$, but the resonance splitting in transmission modulates and vanishes with varying $D$. For an $\mathrm{ABM}$, however, strong coupling can be maintained at all positions inside the cavity. While at $D=0$, the cavity only interacts with the symmetric mode due to the maximum electric field inside the cavity; at $D=0.25 \lambda_{c}$, the cavity only couples to the antisymmetric mode [see Fig. 4(d)]. At intermediate positions, where $0<D<0.25 \lambda_{c}$, the cavity couples to both symmetric and antisymmetric modes so that a spectral splitting persists at all positions. We note that this interesting effect could also be applied to analogous situations involving classical metasurfaces coupled to cavities.

In conclusion, we have demonstrated that synthetic arrangements of quantum emitters with only electric dipole transitions can support both electric and magnetic responses at optical frequencies. Since the interemitter distances required for our proposed designs are well above ten nanometers, our envisaged quantum metasurfaces can 
be experimentally realized in both the gas and solid phases using a range of available methods in cold atom manipulation or implantation strategies. In particular, our proposal lends itself to applications based on natural species with weak magnetic dipole transitions, e.g., rare earth ions, (See Refs $[51,52])$. For instance, $\mathrm{Eu}^{3+}$ with a magnetic transition $\left({ }^{5} D_{0} \rightarrow{ }^{7} F_{1}\right)$ at $584 \mathrm{~nm}$ and a linewidth of about $15 \mathrm{~Hz}$ (see Table III Ref [51]) is a suitable candidate for enhancement by an arrangement of atoms with electric dipole transition at the same wavelength (see Ref. [52], $\mathrm{J}=0 \rightarrow \mathrm{J}=1$ with an E1 transition). The predicted transition rate enhancements reaching $10^{5}$ would, thus, yield magnetic transitions with natural linewidths comparable to that of common electric dipole transitions. Considering the wealth of quantum functionalities such as entanglement or quantum memory accessible to natural emitters such as atoms, novel materials presented here hold promise for the development of a range of technological applications and fundamental studies in quantum engineering and physics.

This work was supported by the Max Planck Society. R. A. is grateful to Peter Milonni for helpful discussions and acknowledges the support of the Alexander von Humboldt Foundation through the Feodor Lynen Fellowship. The authors warmly thank Claudiu Genes for helpful discussions.

*rasoul.alaee@gmail.com

†vahid.sandoghdar@mpl.mpg.de

[1] L. Landau and E. Lifshitz, Electrodynamics of Continuous Media (Elsevier, New York, 2013), Vol. 8, p. 252, Chap. IX, Sec. 60.

[2] J. D. Jackson, Classical Electrodynamics (Wiley, New York, 1999).

[3] V. Sandoghdar, Nano Lett. 20, 4721 (2020).

[4] N. Engheta and R.W. Ziolkowski, Metamaterials: Physics and Engineering Explorations (John Wiley \& Sons, New York, 2006).

[5] C. M. Soukoulis and M. Wegener, Nat. Photonics 5, 523 (2011).

[6] N. Yu and F. Capasso, Nat. Mater. 13, 139 (2014).

[7] A. I. Kuznetsov, A. E. Miroshnichenko, M. L. Brongersma, Y.S. Kivshar, and B. Luk'yanchuk, Science 354, 2472 (2016).

[8] W. Cai and V. M. Shalaev, Optical Metamaterials (Springer, New York, 2010), Vol. 10.

[9] G. Zumofen, N. M. Mojarad, V. Sandoghdar, and M. Agio, Phys. Rev. Lett. 101, 180404 (2008).

[10] V. Sandoghdar, M. Agio, X.-W. Chen, S. Götzinger, and K.-G. Lee, Antennas, quantum optics and near-field microscopy, in Optical Antennas, edited by M. Agio and A. Alù (Cambridge University Press, Cambridge, England, 2013), pp. 100-121.

[11] Z. Meir, O. Schwartz, E. Shahmoon, D. Oron, and R. Ozeri, Phys. Rev. Lett. 113, 193002 (2014).

[12] R. J. Bettles, S. A. Gardiner, and C. S. Adams, Phys. Rev. Lett. 116, 103602 (2016).
[13] E. Shahmoon, D. S. Wild, M. D. Lukin, and S. F. Yelin, Phys. Rev. Lett. 118, 113601 (2017).

[14] M. Zhou, J. Liu, M. A. Kats, and Z. Yu, ACS Photonics 4, 1279 (2017).

[15] V. Mkhitaryan, L. Meng, A. Marini, and F. J. G. de Abajo, Phys. Rev. Lett. 121, 163602 (2018).

[16] D. S. Wild, E. Shahmoon, S. F. Yelin, and M. D. Lukin, Phys. Rev. Lett. 121, 123606 (2018).

[17] M. T. Manzoni, M. Moreno-Cardoner, A. Asenjo-Garcia, J. V. Porto, A. V. Gorshkov, and D. E. Chang, New J. Phys. 20, 083048 (2018).

[18] O. Černotík, A. Dantan, and C. Genes, Phys. Rev. Lett. 122, 243601 (2019).

[19] D. Plankensteiner, C. Sommer, M. Reitz, H. Ritsch, and C. Genes, Phys. Rev. A 99, 043843 (2019).

[20] A. Grankin, P. O. Guimond, D. V. Vasilyev, B. Vermersch, and P. Zoller, Phys. Rev. A 98, 043825 (2018).

[21] I. Liberal, I. Ederra, and R. W. Ziolkowski, Phys. Rev. A 97, 053847 (2018).

[22] P.-O. Guimond, A. Grankin, D. V. Vasilyev, B. Vermersch, and P. Zoller, Phys. Rev. Lett. 122, 093601 (2019).

[23] R. J. Bettles, M. D. Lee, S. A. Gardiner, and J. Ruostekoski, arXiv:1907.07030.

[24] J. Rui, D. Wei, A. Rubio-Abadal, S. Hollerith, J. Zeiher, D. M. Stamper-Kurn, C. Gross, and I. Bloch, Nature (London) 583, 369 (2020).

[25] S. D. Jenkins and J. Ruostekoski, Phys. Rev. A 86, 031602 (R) (2012).

[26] G. Facchinetti and J. Ruostekoski, Phys. Rev. A 97, 023833 (2018).

[27] A. Lagendijk and B. A. Van Tiggelen, Phys. Rep. 270, 143 (1996).

[28] P. Lambropoulos and D. Petrosyan, Fundamentals of Quantum Optics and Quantum Information (Springer, New York, 2007), Vol. 23.

[29] L. L. Foldy, Phys. Rev. 67, 107 (1945).

[30] G. W. Mulholland, C. F. Bohren, and K. A. Fuller, Langmuir 10, 2533 (1994).

[31] See Supplemental Material at http://link.aps.org/supplemental/ 10.1103/PhysRevLett.125.063601 for derivation details of induced multipole moments, scattering cross section of an atomic dimer, the decay rate of a magnetic emitter in the vicinity of an atomic dimer or tetramer, reflection and transmission of an atomic monolayer or bilayer metasurface with and without cavity, which includes Refs. [32-36].

[32] B. E. Saleh and M. C. Teich, Fundamentals of Photonics (Wiley, New York, 1991), Vol. 22.

[33] P. De Vries, D. V. Van Coevorden, and A. Lagendijk, Rev. Mod. Phys. 70, 447 (1998).

[34] L. Novotny and B. Hecht, Principles of Nano-Optics (Cambridge University Press, Cambridge, England, 2012).

[35] A. Alù and N. Engheta, Phys. Rev. B 79, 235412 (2009).

[36] R. Alaee, M. Albooyeh, and C. Rockstuhl, J. Phys. D 50, 503002 (2017).

[37] R. Alaee, C. Rockstuhl, and I. Fernandez-Corbaton, Opt. Commun. 407, 17 (2018).

[38] R. Alaee, C. Rockstuhl, and I. Fernandez-Corbaton, Adv. Opt. Mater. 7, 1800783 (2019).

[39] R. G. DeVoe and R. G. Brewer, Phys. Rev. Lett. 76, 2049 (1996). 
[40] C. Hettich, C. Schmitt, J. Zitzmann, S. Kühn, I. Gerhardt, and V. Sandoghdar, Science 298, 385 (2002).

[41] C.-T. Tai, Dyadic Green Functions in Electromagnetic Theory (Institute of Electrical \& Electronics Engineers (IEEE), New York, 1994).

[42] S. Karaveli and R. Zia, Phys. Rev. Lett. 106, 193004 (2011).

[43] M. Kasperczyk, S. Person, D. Ananias, L. D. Carlos, and L. Novotny, Phys. Rev. Lett. 114, 163903 (2015).

[44] K. Groot-Berning, T. Kornher, G. Jacob, F. Stopp, S. T. Dawkins, R. Kolesov, J. Wrachtrup, K. Singer, and F. Schmidt-Kaler, Phys. Rev. Lett. 123, 106802 (2019).

[45] T. Lühmann, N. Raatz, R. John, M. Lesik, J. Rödiger, M. Portail, D. Wildanger, F. Kleißler, K. Nordlund, A. Zaitsev et al., J. Phys. D 51, 483002 (2018).
[46] M. Endres, H. Bernien, A. Keesling, H. Levine, E. R. Anschuetz, A. Krajenbrink, C. Senko, V. Vuletic, M. Greiner, and M. D. Lukin, Science 354, 1024 (2016).

[47] D. Barredo, S. De Léséleuc, V. Lienhard, T. Lahaye, and A. Browaeys, Science 354, 1021 (2016).

[48] S.-P. Yu, J. A. Muniz, C.-L. Hung, and H. J. Kimble, Proc. Natl. Acad. Sci. U.S.A. 116, 12743 (2019).

[49] S. Tretyakov, Analytical Modeling in Applied Electromagnetics (Artech House, Inc., Norwood, 2003).

[50] F. J. Garcia De Abajo, Rev. Mod. Phys. 79, 1267 (2007).

[51] C. M. Dodson and R. Zia, Phys. Rev. B 86, 125102 (2012).

[52] P. van Hoof, Galaxies 6, 63 (2018). 